\title{
Nuevos modos de gestión institucional en la investigación en Diseño Proyecto de Investigación
}

$\mathrm{N}^{\circ} 8.2$

Equipo de Investigación ${ }^{(1)}$

Marina Matarrese

por Facultad de Diseño y Comunicación, Universidad de Palermo (ARG)

Luz del Carmen Vilchis Esquivel por Universidad Nacional Autónoma de México (MEX)

Resumen: El Proyecto de Investigación 8.2 reflexiona acerca de la compleja relación entre el Diseño y la artesanía, para ello, se agruparon los aportes en dos ejes: el primero desde los aspectos que dan cuenta con profundidad disciplinar y conceptual de los cruces, tensiones, desigualdades y solapamientos que pueden tejerse entre los fundamentos de diseño y las artesanías; también desde las concepciones históricas que fueron forjando el sentido común y disciplinar acerca de los puntos en común y de las divergencias entre los procesos en estudio. En el segundo se reponen parte de estas reflexiones pero atravesadas por las particularidades propias de la producción, creación, circulación, y consumo de los pueblos indígenas en una doble identidad de minoría étnica y artesanal.

Palabras clave: Diseño - Artesanías - Identidad - Desigualdad - Complejidad

[Resúmenes en inglés y portugués en la página 209]

${ }^{(1)}$ Los CVs del Equipo de Investigación pueden consultarse en el Capítulo Directores de Líneas y Coordinadores de Proyectos de esta misma Edición.

\section{Acerca del Proyecto 8.2}

Nuevos modos de gestión institucional en la Investigación en Diseño

El Proyecto 8.2 Nuevos modos de gestión institucional en la Investigación en Diseño, presenta y se introduce en la exploración comparada y contribución al desarrollo del campo teórico y metodológico de la investigación en diseño y comunicación. Se articula con el Programa de Investigación para impulsar y fortalecer investigaciones que impacten en el desarrollo futuro de las disciplinas, tanto a nivel académico como profesional. 
Se acordó entre la Universidad de Palermo y la Universidad Nacional de México, avanzar en una investigación en la que participan académicos de ambas Instituciones, con la coordinación compartida de Marina Matarrese (UP) y Luz del Carmen Vilchis Esquivel (UNAM), con el fin de impactar en las estrategias de investigación en diseño en Latinoamérica, a través de comparar las diversas experiencias realizadas en Instituciones de Educación Superior de la región con la llevada adelante por la Facultad de Diseño y Comunicación de la Universidad de Palermo. Las reflexiones y los resultados obtenidos en el Proyecto 8.2 Nuevos modos de gestión institucional en la investigación en Diseño continúa los obtenidos en el 8.1 Experiencias comparadas: Investigar Diseño en América Latina y se continúan desarrollando en el Proyecto 8.3 Identidad, Ética y Diseño: Un análisis de los múltiples cruces posibles, en el marco de la misma Línea de Investigación y bajo la misma Directora Marina Matarrese.

Sus principales objetivos son:

- Analizar el complejo y multívoco diálogo entre el Diseño y las artesanías a partir de diversos casos de estudio.

- Indagar acerca del concepto de Artesanía como proceso y las instancias de comercialización.

- Mapear diversos casos de estudios en los que confluyen las artesanías con el Diseño.

- Teorizar acerca de las diferencias conceptuales entre Artesanías y Diseño.

\section{Acerca de la Línea 8}

Investigar en Diseño dirigida por Marina Matarrese se desarrolla de manera ininterrumpida desde 2015 en la Facultad de Diseño y Comunicación (UP, Argentina), e incluye hasta el momento tres proyectos finalizados el 8.1 Experiencias comparadas: Investigar Diseño en América Latina, el 8.2 Nuevos modos de gestión institucional en la investigación en Diseño, y el 8.3 Identidad, Ética y Diseño: Un análisis de los múltiples cruces posibles, coordinados por Marina Matarrese (UP) y Luz del Carmen Vilchis Esquivel (UNAM).

\section{Mapa de Áreas y Proyectos}

El Proyecto 8.2 se vincula con todas las carreras de grado y posgrado correspondientes a la Facultad de Diseño y Comunicación.

\section{Productos y Resultados}

\section{a)- Publicaciones}

Cuaderno del Centro de Estudios de Diseño y Comunicación No90. (2021) Investigar en diseño: del complejo y multívoco diálogo entre Diseño y las artesanías. Coordinación 
Marina Matarrese. Año XXII, Abril 2021, Buenos Aires, Argentina. ISSN: 1668-0227. Esta publicación documenta y comunica los resultados alcanzados en el proyecto de investigación 8.2 Nuevos modos de gestión institucional en la investigación en diseño, y a continuación se detallan los autores y artículos contenidos en ella:

Matarrese Marina Laura y Vilchis Esquivel Luz del Carmen (2021) Prólogo (Pp. 11 a 19)

Vilchis Esquivel Luz del Carmen (2021) Del símbolo a la trama (Pp. 21 a 34) Garrido María Laura (2021) La división arte/artesanía y su relación con la construcción de una historia del diseño (Pp. 35 a 46)

Sandoval Valle Marco Antonio (2021) Relaciones de complejidad e identidad entre artesanía y diseño (Pp. 47 a 59)

Rubio Toledo Miguel Angel (2021) El Diseño sistémico transdisciplinar para el desarrollo sostenible neguentrópico de la producción artesanal (Pp. 61 a 75)

del Moral Zamudio, Yésica A. (2021) La innovación en la creación y comercialización de animales fantásticos en Arrazola, Oaxaca (Pp. 77 a 89)

De La Barrera Medina, Mónica Susana (2021) El diseño como objeto artesanal de consumo e identidad (Pp. 91 a 106)

Trocha Paola (2021) Las artesanías Zenú: transformaciones y continuidades como parte de diversas estrategias artesanales (Pp. 107 a 124)

Martínez González Mercedes, García García Fernando (2021) El espejo en que nos vemos juntos: la antropología y el diseño en la creación de un video mapping arquitectónico con una comunidad purépecha de México (Pp. 125 a 144)

Arévalo Ortiz Paolo (2021) La cultura visual en el proceso del tejido Puruhá (Pp. 145 a 159)

Larrea Solórzano Daniela (2021) La artesanía salasaca y sus procesos de transculturación estética (Pp. 161 a 174)

Ponce Pérez Annabella y Cornejo Ramón Carolina (2021) El diseño textil como resultado de la interacción étnica en Quito, a finales del siglo XVIII (Pp. 175 a 191) Ariza Verónica y Andrade Mar Itzel (2021) La relación artesanía y diseño. Estudios desde el norte de México (Pp. 193 a 211)

Alvarez Saavedra, Eugenia (2021) El diseño representado a través de la artesanía. Emprendedores de la etnia Mapuche. Región de la Araucanía, Chile (Pp. 213 a 225).

Actas de Diseño No30. (2020) XIV Encuentro Latinoamericano de Diseño "Diseño en Palermo" X Congreso Latinoamericano de Enseñanza del Diseño Comunicaciones Académicas. Buenos Aires, Argentina. Se documentan las ponencias de la Comisión Investigar en Diseño: Proyecto 8.2 Nuevos modos de gestión institucional en la investigación en diseño correspondiente a la presentación del Cuaderno 90 durante el IV Coloquio de Investigación y Desarrollo en Diseño (Ponencias en Pp. 46-47).

Actas de Diseño No27. (2019) XIII Encuentro Latinoamericano de Diseño "Diseño en Palermo" IX Congreso Latinoamericano de Enseñanza del Diseño Comunicaciones Académicas. Facultad de Diseño y Comunicación, Universidad de Palermo. Buenos Aires, 
Argentina. Se documentan las ponencias de la Comisión Investigar en Diseño: Proyecto 8.2 Nuevos modos de gestión institucional en la investigación en diseño correspondientes a la presentación del Cuaderno 90 durante el III Coloquio de Investigación y Desarrollo en Diseño (Ponencias en Pp. 52-53), y Proyecto 8.1 Experiencias comparadas: Investigar Diseño en América Latina, en el II Coloquio de Investigación y Desarrollo en Diseño (Pp.12).

\section{b)- Congresos / Coloquios / Plenarios}

$3^{\circ}$ Plenario de Directores de Investigación DC. 26, 27 y 29 de agosto de 2019. En este tercer plenario los Directores de las Líneas y Proyectos de Investigación presentaron a sus pares y al conjunto del Programa de Investigación de la Facultad de Diseño y Comunicación, los resultados obtenidos y/o en proceso (publicaciones y acuerdos), junto con los avances de Proyectos. La Directora de la Línea de Investigación No1 presentó a sus pares, los resultados del Proyecto 8.2 Nuevos modos de gestión institucional en la investigación del Diseño y anticipó los nuevos avances del cuarto proyecto 8.3 Identidad, Ética y Diseño. Un Análisis de los múltiples cruces posibles (en curso).

IV Coloquio de Investigación y Desarrollo en Diseño Latino. Universidad de Palermo, 29 de julio de 2019. X Congreso Latinoamericano de Enseñanza del Diseño. Semana Internacional del Diseño en Palermo.

En la comisión Investigar Diseño se presentaron las reflexiones y conclusiones del Proyecto de Investigación 8.2 Nuevos modos de gestión institucional en la investigación en diseño, coordinado por Marina Matarrese (UP) y Luz del Carmen Vilchis (UNAM) pertenecientes al Cuaderno 90 Diálogos entre el diseño, la artesanía y la identidad. Se debatió acerca del marco del ejercicio del diseño, en tanto compleja urdimbre cuyos objetos de análisis abarcan tanto sus condiciones de desarrollo, las influencias del pensamiento estético y las coyunturas socioculturales en las que se inscriben tanto los diseñadores, las políticas orientadas al diseño cuanto los objetos diseñados con la industria y los artesanales. Expusieron: María Laura Garrido, Mercedes González, Eugenia Álvarez Saavedra, Anabella Ponce.

A continuación se detallan las ponencias presentadas en esta Comisión coordinada por la Directora del proyecto Marina Matarrese:

María Laura Garrido (Argentina)

La división arte/artesanía y su relación con la construcción de una historia del diseño Mercedes González (México)

La antropología y el diseño: una propuesta de video mapping colaborativo

Eugenia Álvarez Saavedra (Chile)

El diseño representado a través de la artesanía. Emprendedores de la etnia Mapuche. Región de la Araucanía, Chile

Anabella Ponce (Ecuador)

El diseño textil como resultado de la interacción étnica en Quito, a finales del siglo VIII 
Juan Carlos Morales Posada (Colombia)

Representación, retórica y estética en la visualización científica. (Socialización de avances del proyecto de doctorado)

Mónica Susana De La Barrera Medina (México)

Reflexiones acerca de investigaciones en diseño en relación con las artesanías

Luz del Carmen Vilchis Esquivel (México)

Preguntar al diseño es Investigar el diseño

\title{
c)- Formación de Posgrado e Impacto curricular
}

La Directora Marina Matarrese es Docente de Posgrado en Diseño de la Facultad de Diseño y Comunicación en Maestría en Gestión del Diseño y Doctorado en Diseño y al igual que Luz del Carmen Vilchis Esquivel en su institución (UNAM), incorporan los contenidos de su investigación a sus asignaturas en posgrado.

\begin{abstract}
Research Project 8.2 reflects on the complex relationship between Design and craftsmanship, for this, the contributions were grouped into two axes: the first from the aspects that account with disciplinary and conceptual depth of the crossings, tensions, inequalities and overlaps that can be woven between the fundamentals of design and crafts; also from the historical conceptions that were forging common and disciplinary sense about the common points and the divergences between the processes under study. In the second part of these reflections are replaced but crossed by the particularities of the production, creation, circulation, and consumption of indigenous peoples in a double identity of ethnic and artisanal minority.
\end{abstract}

Keywords: Design - Crafts - Identity - Inequality - Complexity

Resumo: O Projeto de Pesquisa 8.2 reflete sobre a complexa relação entre Design e artesanato, para isso, as contribuições foram agrupadas em dois eixos: o primeiro a partir dos aspectos que dão conta da profundidade disciplinar e conceitual dos cruzamentos, tensões, desigualdades e sobreposições que podem ser tecidas entre os fundamentos do design e do artesanato; também a partir das concepções históricas que foram forjando o senso comum e disciplinar sobre os pontos comuns e as divergências entre os processos em estudo. Na segunda parte, essas reflexões são substituídas mas percorridas pelas particularidades da produção, criação, circulação e consumo dos povos indígenas em uma dupla identidade de minoria étnica e artesanal.

Palavras chave: Design - Artesanato - Identidade - Desigualdade - Complexidade

[Las traducciones de los resúmenes fueron realizadas a través de traductor automático] 